\title{
Clinical practice guidelines and the overuse of health care services: need for reform
}

\author{
Ismail Jatoi MD PhD, Sunita Sah MD PhD
}

Cite as: CMAJ 2019 March 18;191:E297-8. doi: 10.1503/cmaj.181496

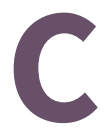

linical practice guidelines can enhance the delivery of evidence-based health care. Yet, after reviewing the same evidence, at about the same time and from the same sets of clinical trials, medical panels in different parts of the world have issued varying, and sometimes even conflicting, guidelines. Guidelines issued by medical specialty societies in North America are often at odds with European guidelines and those of independent North American organizations that have few or no vested financial interests in the medical services at stake. ${ }^{1,2}$ Variability among guidelines raises concerns about validity and may reflect the biases of panellists from different cultural and clinical settings (for example, the United States has a largely fee-for-service health care system and a more litigious culture than most countries). Regardless of country of origin, physicians often recommend procedures and treatments that they are trained to provide, a phenomenon known as "specialty bias." This may explain why medical specialty societies frequently issue guidelines calling for greater use of health care services linked to their specialties (Appendix 1, available at www.cmaj.ca/lookup/ suppl/doi:10.1503/cmaj.181496/-/DC1), thereby exacerbating overdiagnosis, overtreatment and increasing health care costs.

In 2011, the US Institute of Medicine proposed standards for developing rigorous and trustworthy clinical practice guidelines. ${ }^{4}$ It called for the creation of multidisciplinary guideline panels and urged panellists to disclose services from which they derive a substantial portion of their income (i.e., fee-for-service conflicts of interest). These recommendations appear to have had little impact.

In 2016, the Canadian Association of Radiologists issued a guideline calling for women with average breast cancer risk to begin screening mammography at age 40 (https://car.ca/wp -content/uploads/Breast-Imaging-and-Intervention-2016.pdf). By contrast, the Canadian Task Force on Preventive Health Care and many European organizations, including the European Society for Medical Oncology, recommend that screening begin at age $50 .{ }^{5,6}$ The discrepancy could be attributable to specialty bias, fee-for-service conflicts of interest, or both. Notably, 7 of the 8 members of the committee for the recent Canadian Association of Radiologists guideline were radiologists, as compared with none of the 10 Canadian task force panellists, and 1 out of

\section{KEY POINTS}

- Specialty bias and fee-for-service conflicts of interest threaten the validity of clinical practice guidelines and may lead to overdiagnosis, overtreatment and increasing health care costs.

- Clinical practice guidelines issued by medical specialty societies in North America frequently call for greater use of health care services linked to their specialties and are often at odds with European guidelines and those of independent North American organizations.

- Clinical practice guideline panels should be multidisciplinary in composition, independent of the governing bodies of medical specialty societies, and strive to reduce fee-for-service conflicts of interest.

8 European Society for Medical Oncology panellists. Since the 1980 s, screening mammography has been widely recommended from age 40 onward in the US, but declines in breast cancer mortality in the US mirrored those of western Europe, where screening generally begins at age 50 years, suggesting that more aggressive screening does not improve patient outcomes. ${ }^{\text {? }}$

In the US, the National Comprehensive Cancer Network, an alliance of cancer centres, included 25 urologists on its 32-member panel for the prostate cancer screening guideline and recommended screening with prostate-specific antigen (PSA) starting at age 45 years, and beyond age 75 in healthy men with few or no comorbidities. ${ }^{2}$ However, the Canadian task force and European Society for Medical Oncology recommend against PSA screening for men of all ages. ${ }^{2}$ Given that a positive PSA test result generates further urological interventions, a process known as "investigation momentum," the National Comprehensive Cancer Network recommendations may again be attributable to specialty bias, fee-for-service conflicts of interest, or both. ${ }^{8}$ It is noteworthy that none of the 9 Canadian task force panel members and only 1 of the 4 European Society of Medical Oncology panellists were urologists. However, a more litigious culture in the US may also have influenced the National Comprehensive Cancer Network to recommend PSA screening. In the US, mortality rates from prostate cancer declined within 10 years after implementation of PSA screening, but similar 
mortality trends were also reported in the United Kingdom, where PSA screening was discouraged. ${ }^{9}$

When several options are available, medical specialty societies may favour procedures and interventions linked to their specialties. For example, a guideline developed by a 6-member panel of the American College of Gastroenterology, all of whom were gastroenterologists, recommended colonoscopy as the preferred screening strategy for colorectal cancer. ${ }^{3}$ In contrast, the United States Preventive Services Task Force, with no gastroenterologists or gastrointestinal surgeons on its 16 -member panel, recommended screening with fecal occult blood testing, sigmoidoscopy or colonoscopy. ${ }^{3}$ The 7 -member European Society for Medical Oncology guideline panel, which consisted of 6 medical oncologists and 1 gastrointestinal surgeon, recommended fecal occult blood testing, and stated there was limited evidence that screening colonoscopy is effective. ${ }^{3}$ Colonoscopies are more invasive than fecal occult blood testing and come with potentially greater risks and costs for patients - but increased clinical volume and profits for gastroenterologists.

Finally, the American College of Cardiology/American Heart Association (ACC/AHA) has recommended that prophylactic carotid artery stenting be considered in highly selected patients with asymptomatic carotid stenosis. ${ }^{10}$ After reviewing the same evidence, the UK's National Institute for Health and Care Excellence (NICE) came to the opposite conclusion: that the evidence to support carotid artery stenting was inadequate. ${ }^{10}$ Of the 22 members of the ACC/AHA task force, 18 were cardiologists, while the 5 members of the NICE guideline panel consisted of 2 neuroradiologists, 2 interventional radiologists and 1 vascular surgeon (all specialists with training in carotid artery stenting). This time, both panels were composed predominantly of specialists with expertise in this type of stenting, but the ACC/AHA specialists practised in a largely fee-for-service health care system, while the NICE specialists practised in the UK's National Health Service. Thus, even though specialists may see a more severe disease spectrum than generalists, this alone cannot account for their inclination to recommend more intensive diagnostic and treatment guidelines.

Evidence-based clinical practice guidelines can improve health care delivery. Yet specialty bias and fee-for-service conflicts of interest threaten their validity and may lead to unnecessary overuse of health care services. More is not necessarily better in medicine; if anything, patient outcomes may be worse the more "care" they receive. ${ }^{11}$ Every medical test, procedure and treatment adds risk against potential benefit, and some may lead to more harm than good.
Although disclosure alone is not a panacea for addressing specialty bias and fee-for-service conflicts of interest, we believe it is an important first step.,4 The Guidelines International Network suggests that health care professionals with conflicts of interest serve as external advisers rather than voting members of guideline panels, and this proposal merits further consideration. ${ }^{12}$ Moreover, medical specialty societies should acknowledge that they serve the interests of their memberships, which are not always aligned with the public's interests. Guideline panels should be multidisciplinary in composition, independent of the governing bodies of specialty societies, and aim to reduce fee-for-service conflicts of interest. ${ }^{4}$ Panels that comprise only specialists may lack sufficient heterogeneity and potential for voicing dissenting opinions. Guideline panels should ideally contain individuals with expertise in epidemiology, biostatistics and clinical trial methodology, along the lines of those assembled by the Canadian task force, United States Preventive Services Task Force and NICE. Specialty societies should do the same lest guidelines from independent bodies become the only ones deemed to meet adequate standards.

\section{References}

1. Bénard F, Barkun AN, Martel M, et al. Systematic review of colorectal cancer screening guidelines for average-risk adults: Summarizing the current global recommendations. World J Gastroenterol 2018;24:124-38.

2. Pinsky PF, Prorok PC, Kramer BS. Prostate cancer screening - a perspective on the current state of the evidence. N Engl J Med 2017;376:1285-9.

3. Sah S, Fagerlin A, Ubel P. Effect of physician disclosure of specialty bias on patient trust and treatment choice. Proc Natl Acad Sci U S A 2016;113:7465-9.

4. Institute of Medicine (US) Committee on Standards for Developing Trustworthy Clinical Practice Guidelines. In: Graham R, Mancher M, Miller Wolman D, et al., editors. Clinical practice guidelines we can trust. Washington (DC): National Academies Press; 2011.

5. Klarenbach S, Sims-Jones N, Lewin G, et al. Recommendations on screening for breast cancer in women aged 40-74 years who are not at increased risk for breast cancer. CMAJ 2018;190:E1441-51.

6. Senkus E, Kyriakides S, Ohno S, et al. Primary breast cancer: ESMO clinical practice guidelines for diagnosis, treatment and follow-up. Ann Oncol 2015;26 (Suppl 5):v8-30.

7. Jatoi I, Miller AB. Why is breast-cancer mortality declining? Lancet Oncol 2003;4:251-4.

8. Sah S, Elias P, Ariely D. Investigation momentum: the relentless pursuit to resolve uncertainty. JAMA Intern Med 2013;173:932-3.

9. Oliver SE, Gunnell D, Donovan JL. Comparison of trends in prostate-cancer mortality in England and Wales and the USA. Lancet 2000;355:1788-9.

10. Paraskevas KI, Mikhailidis DP, Veith FJ. Comparison of the five 2011 guidelines for the treatment of carotid stenosis. J Vasc Surg 2012;55:1504-8.

11. Morgan DJ, Dhruva SS, Wright SM, et al. 2016 update on medical overuse: a systematic review. JAMA Intern Med 2016;176:1687-92.

12. Schünemann HJ, Al-Ansary LA, Forland F, et al. Guidelines International Network: principles for disclosure of interests and management of conflicts in guidelines. Ann Intern Med 2015;163:548-53.

\section{Competing interests: None declared.}

This article has been peer reviewed.

Affiliations: Division of Surgical Oncology and Endocrine Surgery (Jatoi), University of Texas Health, San Antonio, Tex.; Samuel Curtis Johnson Graduate School of Management (Sah), Cornell SC Johnson College of Business, Cornell University, Ithaca, NY
Contributors: Both authors contributed to the conception and design of the work, drafted the manuscript, revised it critically for important intellectual content, gave final approval of the version to be published and agreed to be accountable for all aspects of the work.

Correspondence to: Ismail Jatoi, jatoi@uthscsa.edu 